\title{
Spatial Distribution of Nanocrystals Imaged at the Liquid-Air Interface
}

\author{
Jos van Rijssel, ${ }^{1}$ Marte van der Linden, ${ }^{1}$ Johannes D. Meeldijk, ${ }^{2}$ Relinde J. A. van Dijk-Moes, ${ }^{3}$ \\ Albert P. Philipse, ${ }^{1}$ and Ben H. Erné ${ }^{1, *}$ \\ ${ }^{1}$ Van 't Hoff Laboratory for Physical and Colloid Chemistry, Padualaan 8, 3584 CH, Utrecht, Netherlands \\ ${ }^{2}$ Electron Microscopy Utrecht, Padualaan 8, 3584 CH, Utrecht, Netherlands \\ ${ }^{3}$ Condensed Matter and Interfaces, Princetonplein 1, 3584 CC, Utrecht, Netherlands \\ (Received 14 December 2012; revised manuscript received 3 June 2013; published 3 September 2013)
}

The 3D distribution of nanocrystals at the liquid-air interface is imaged for the first time on a singleparticle level by cryogenic electron tomography, revealing the equilibrium concentration profile from the interface to the bulk of the liquid. When the surface tension of the liquid is decreased, the interaction of the nanocrystals with the liquid-air interface shifts from adsorption to desorption. Macroscopic surface tension measurements do not detect this transition, due to the presence of surface-active molecular species.

DOI: 10.1103/PhysRevLett.111.108302

PACS numbers: 82.70.Dd, 68.37.Lp, 68.03.Cd

Adsorption of colloidal particles at liquid interfaces is a versatile way to control the stability of emulsion droplets or microbubbles [1-4] or to guide the self-assembly of colloidal particles into superlattices or membranes [5-7]. For particles of only a few nanometers in size, adsorption to the liquid-gas or liquid-liquid interface can be of order $k_{B} T$ and reversible [8], as for molecular surfactants. To evaluate how strongly nanoparticles adsorb to liquid interfaces, the conventional method is to measure the interfacial tension as a function of particle concentration [9-12]. However, in the presence of surface-active molecular species, their adsorption will dominate the changes in macroscopic interfacial tension and obscure the effect of adsorbing colloidal nanoparticles. Here, we introduce cryogenic electron tomography as a promising tool to investigate the adsorption of nanoparticles at the liquidair interface. This technique resolves the spatial positions of individual nanoparticles, quantifying the complete concentration profile from the interface to the bulk of the liquid. From the profile, the adsorption energy is calculated and compared to the energies of the interfaces separating solid, liquid, and air.

Previous in situ characterizations did not provide a direct visualization of the distributions of individual nanoparticles at the liquid-air interface but instead relied on macroscopic measurements of the interfacial tension [12] or grazing incidence x-ray scattering [13]. Single-particle techniques like atomic force microscopy [14] do not detect the nanoparticles present below the interface or have insufficient resolution to resolve particles of a few nanometers, as in the case of scanning electron microscopy [15]. The technique that we adopt here, cryogenic electron microscopy (CEM), is well known in biology [16] and gives snapshots even of structures that only exist in a narrow temperature window, such as the molecular liquid crystalline phases of a lipid membrane [17]. Also, in the field of materials science, CEM is becoming an established way to visualize fragile structures at the nanoscale [18]. We have introduced CEM before to image nanoparticle structures in colloidal dispersions, be it only in two dimensions [19-22]. Here, the sample structure is obtained in three dimensions using tomography [23].

Our experimental model system consists of PbSe nanocrystals with a diameter of $5.3 \mathrm{~nm}$, dispersed in mixtures of decalin and pentanol. The so-called quantum dots are synthesized according to a variation on the method of Heiss et al. [24] (see the Supplemental Material [25] for details). The surface of the particles is covered with oleic acid ligands, in order to disperse the particles in an apolar liquid. In a thermostatted Vitrobot, $3 \mu \mathrm{l}$ of $0.4 \mu \mathrm{mol} / 1$ quantum dot dispersion is placed on a QuantiFoil TEM grid with holes of $2 \mu \mathrm{m}$. Excess fluid is removed using absorbent paper, resulting in a film with a thickness of a few particle diameters, after which the sample is vitrified using liquid nitrogen. For each sample, typically three tilt series of the vitrified samples are acquired on a Philips Tecnai 20 transmission electron microscope (200 keV) making use of a liquid-nitrogen-cooled Gatan 626 hightilt cryoholder. The tomograms are reconstructed using the weighted back projection implemented in IMOD [26] from tilt series containing approximately 100 images acquired at angles between $-65^{\circ}$ and $65^{\circ}$.

Our cryogenic electron tomography results are summarized in Fig. 1. Representative $X-Z$ views of samples with different volume fractions of pentanol are shown in Figs. 1(a)-1(c), where the $Z$ axis is perpendicular to the film. From the tomograms, the particle positions were detected, as shown in Figs. 1(d)-1(f), using regular particle tracking algorithms implemented in MATLAB 7. In tomography, contrast variations in the $Z$ direction are hard to resolve due to the limits of the tilt range. Nevertheless, due to density fluctuations in the bulk liquid, a clear difference in background noise is observed between liquid and vacuum, which makes it possible to resolve the position of the 

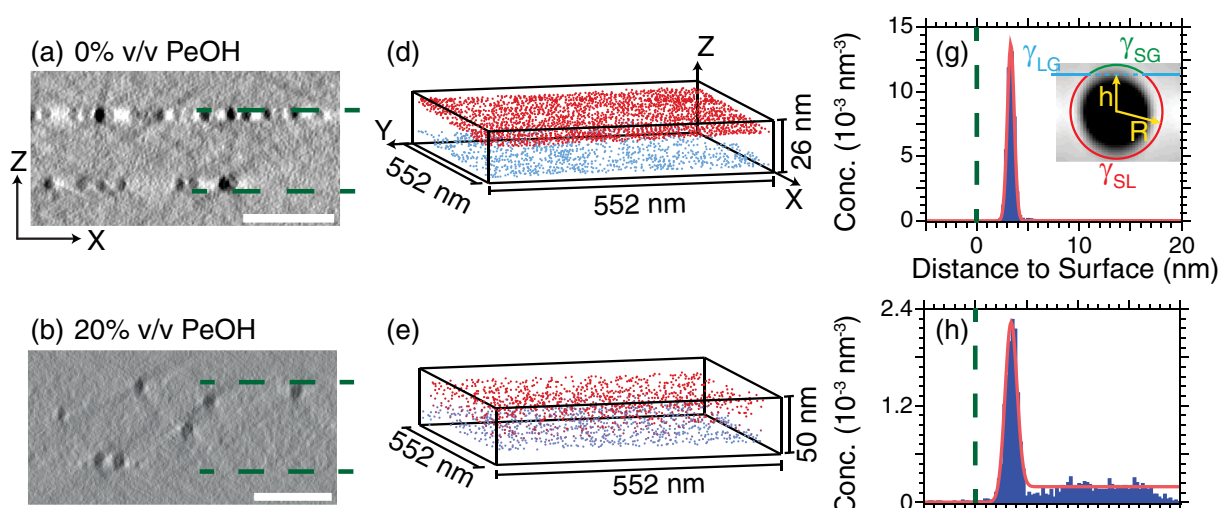

(e)

(c) $25 \% \mathrm{v} / \mathrm{v} \mathrm{PeOH}$
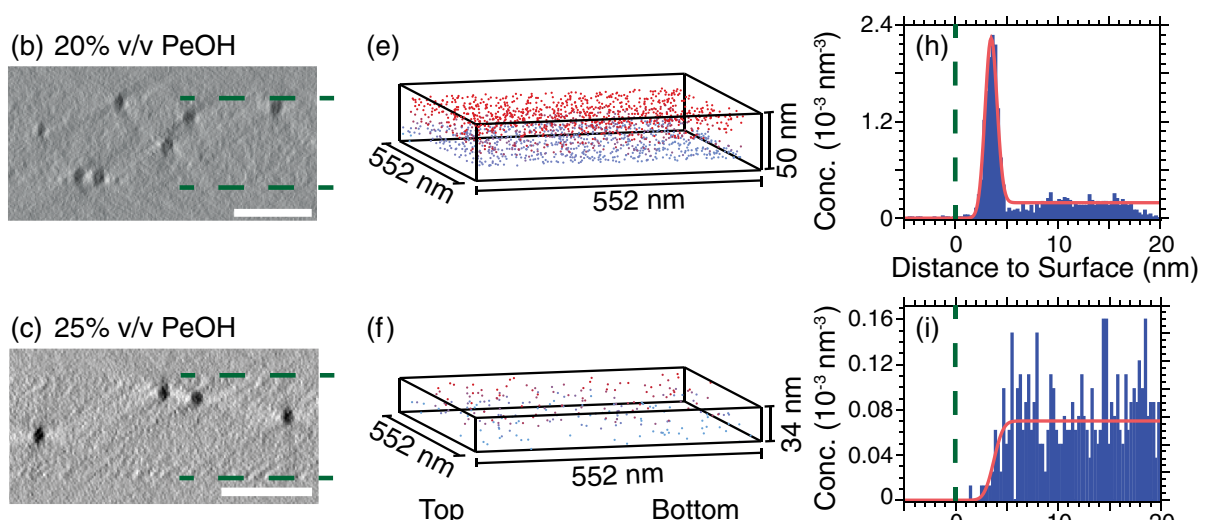

(f)

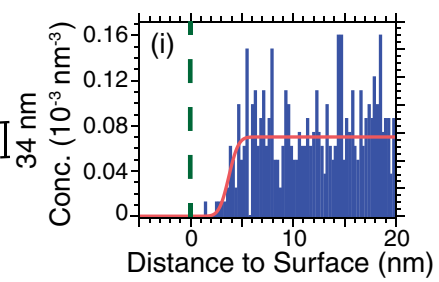

FIG. 1 (color online). Cryogenic transmission electron tomography of freestanding thin liquid films of decalin-pentanol (PeOH) mixtures with added PbSe nanoparticles. (a)-(c) Representative cross sections perpendicular to the film with white scale bars of $25 \mathrm{~nm}$, (d)-(f) 3D plots of the positions of the detected particles (movies of the data sets are provided in the Supplemental Material [25]), and (g)-(i) concentrations in planes parallel to the film as a function of distance to the interface. The nanoparticles have a much stronger affinity to the surface of decalin than to decalin-pentanol mixtures, and surface coverage clearly remains less than a monolayer in all cases. The inset in (g) shows a close-up of a nanoparticle (average image of 100 nanoparticles) of radius $R$ at a distance $h$ from of the decalin-air interface, with the interfacial tensions $(\gamma)$ between liquid $(L)$, particle $(S)$, and air $(G)$.

liquid-air interfaces from spatial analysis of the intensity of the noise. Using this information, it is straightforward to calculate the distance of a particle center to the nearest interface [Figs. 1(g)-1(i)], which reveals the equilibrium distribution of particles between interface and bulk of the liquid. From Fig. 1, it is clear that the equilibrium distribution shifts from strong adsorption, when the apolar liquid decalin is used [Fig. $1(\mathrm{~g})$ ], to weak adsorption, when the polar liquid pentanol is added [Fig. 1(i)].

It is well known that the addition of a polar liquid such as methanol can induce cluster formation and (size selective) precipitation of the nanoparticles [27]. However, larger alcohols such as butanol [27] and pentanol have this effect to a lesser extent. In pure decalin, the clustering of these particles is reversible, with a coupling free energy of approximately $-2 k_{B} T$ [22], indicating that only a small fraction of the particles is present as a cluster; these clusters are constantly forming and breaking up and therefore can adjust their shapes to the energy potential set by the interface. Although the aggregation strength increases with increasing pentanol concentration, the sample precipitates only at concentrations above 63\% (see Fig. S2 in the Supplemental Material [25]). Below 63\% v/v pentanol, the samples are stable for at least one week, indicating that the interactions are weak, even at much higher concentrations than the highest pentanol concentration used in our measurements $(25 \% v / v)$. This indicates that the distributions observed with cryogenic electron tomography are mainly dictated by the adsorption of the particles to the liquid-air interface.

The conventional way to quantify reversible adsorption to the liquid-air interface is less direct. It relies on measurement of the macroscopic surface tension as a function of the concentration of the surface-active species. We measured the macroscopic surface tension of our liquid dispersions with a Dataphysics OCA15 pendant drop tensiometer. From thermodynamics, the adsorption density $\Gamma$ can be calculated using the Gibbs adsorption equation [28]. The common analysis assumes that besides pure solvent, the only other chemical component is the adsorbing species, so that

$$
\Gamma=-\partial \gamma / \partial \mu=-\left(1 / k_{B} T\right) \partial \gamma / \partial \ln (\phi),
$$

where $\gamma$ is the macroscopic interfacial tension and $\mu$ the chemical potential of the adsorbing species, for ideal particles defined as $\mu=\mu^{0}+k_{B} T \ln (\phi)$, with $k_{B}$ Boltzmann's constant, $T$ the absolute temperature, and $\phi$ the volume fraction of the particles. A more general form of the Gibbs adsorption equation is $d \gamma=\sum_{i} \Gamma_{i} d \mu_{i}$, for a system with different components $i$ [28]. In our case, the liquid consists of two components when decalin and pentanol are mixed, but their chemical potentials hardly change when the nanocrystals are added, since the particle volume fraction remains below 0.01; as such, Eq. (1) remains a good first 


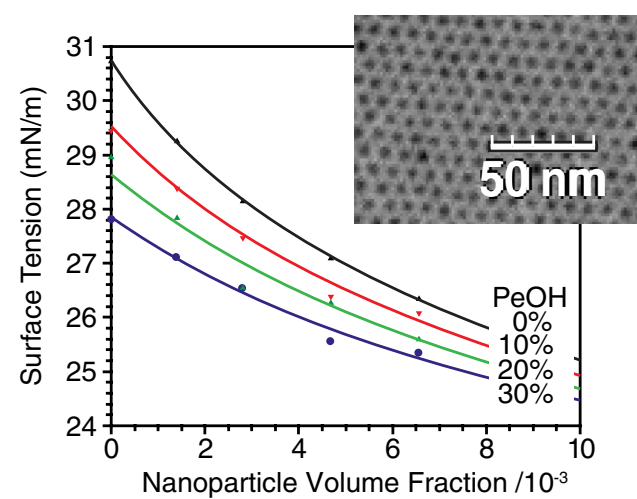

FIG. 2 (color online). Interfacial tension of the liquid-air interface for mixtures of decalin and pentanol $(\mathrm{PeOH})$ as a function of the concentration of PbSe nanoparticles. Inset: Cryogenic transmission electron microscopy image of a full monolayer of particles, indicating the maximum possible packing density.

approximation. As seen in Fig. 2, in the absence of nanoparticles, the addition of pentanol decreases the interfacial tension. With increasing nanoparticle concentration, the interfacial tension decreases further gradually, indicating reversible adsorption. The slope of $\gamma$ versus $\ln \phi$ is more or less the same, regardless of the pentanol concentration. Applying Eq. (1), we find an adsorption density of $4 \times 10^{17} \mathrm{~m}^{-2}$, while electron microscopy shows that a close-packed monolayer can accommodate only $0.12 \times 10^{17} \mathrm{~m}^{-2}$ particles (see the inset of Fig. 2).

Rather than helping to explain the adsorption behavior observed by cryogenic electron tomography, the surface tension measurements are in conflict with it. The macroscopic surface tension suggests almost complete independence of the adsorption density on the decalin-pentanol ratio, whereas the tomographic measurements show a transition from strong adsorption to complete desorption. To resolve this discrepancy, we will first explain the adsorption behavior observed with cryogenic tomography on the basis of different contributions to the adsorption free energy of a nanoparticle at the liquid-air interface. The apparently unrelated behavior of the macroscopic surface tension will then be explained separately.

The concentration profiles as obtained from cryogenic electron tomography can be accounted for as follows. For an equilibrium system, the number density $\rho$ of the particles at a distance $h$ from the particle center to the liquid-air interface depends on the adsorption free energy $F(h)$, according to

$$
\rho(h)=\rho(\infty) \exp \left[-F(h) /\left(k_{B} T\right)\right],
$$

where $\rho(\infty)$ is the number density in the bulk liquid. The free energy profile is estimated via the Pieranski [29] approach, which has been successfully applied to many microparticle systems [1]. For a particle of radius $R$ at a distance $|h|<R$ from the liquid-air interface [see the inset in Fig. 1(g)], the interfacial energy can be described by the sum of two contributions. First, the surface area of the liquid-air interface is decreased as the particle sticks out, resulting in a decrease in free energy of $\Delta F=$ $-\gamma_{L G} \pi\left(R^{2}-h^{2}\right)$, where $\gamma_{L G}$ is the liquid-air interfacial tension. Second, part of the particle surface is now exposed to the gas, resulting in a change in free energy of $\Delta F=$ $\left(\gamma_{S G}-\gamma_{S L}\right) 2 \pi R^{2}(1-h / R)$, where $\gamma_{S G}$ and $\gamma_{S L}$ are the interfacial tensions of the particle surface in, respectively, the gas or the liquid. Characterization of the binding strength of colloidal particles at a liquid-air interface is typically done by the contact angle $\theta$, defined as $\cos (\theta)=$ $\Delta \gamma / \gamma_{L G}=\left(\gamma_{S G}-\gamma_{S L}\right) / \gamma_{L G}$. In Figs. 1(g)-1(i), the solid red curve indicates the fitted distribution as obtained with this model using $\Delta \gamma$ and $\rho(\infty)$ as the fitting parameters and taking $\gamma_{L G}$ from surface tension measurements on pure solvent mixtures: $30.7 \mathrm{mN} / \mathrm{m}$ for pure decalin, $28.7 \mathrm{mN} / \mathrm{m}$ for $20 \% v / v$ pentanol, and $28.3 \mathrm{mN} / \mathrm{m}$ for $25 \% v / v$ pentanol. To take into account the experimental resolution and fluctuations in the position of the interface, the distribution is broadened with a Gaussian having a standard deviation of $0.3-0.6 \mathrm{~nm}$, corresponding to $1-2$ pixels. The description using Eq. (2) and a potential energy in terms of $\gamma_{L G}, \gamma_{L S}$, and $\gamma_{S G}$ is clearly sufficient for a good fit of all the profiles.

Quite high values of $\Delta \gamma=\gamma_{S G}-\gamma_{S L}$ were obtained from the fit: in the case of pure decalin as the liquid, $\Delta \gamma=$ $25.9 \mathrm{mN} / \mathrm{m}$, corresponding to a contact angle of $32^{\circ}$, and in the case of $20 \% v / v$ pentanol, $\Delta \gamma=25.7 \mathrm{mN} / \mathrm{m}$ and $\theta=26^{\circ}$. With $25 \% v / v$ pentanol, the particles do not adsorb at all, so the contact angle is not defined, and only a lower bound to $\Delta \gamma$ can be given: $\Delta \gamma>\gamma_{L G}=$ $28.3 \mathrm{mN} / \mathrm{m}$. The fact that $\Delta \gamma$ has a high value can be understood on the basis of the surface coverage of the $\mathrm{PbSe}$ nanoparticles with oleic acid molecules; they consist of a carboxylic acid head group bound to the nanoparticle surface and an apolar octadecene tail pointing away from the nanoparticle. The surface of the nanoparticle is thus similar to octadecene (OD), and the particle-air interfacial tension is approximately that of octadecene-air tension $\gamma_{S G} \cong \gamma_{\mathrm{OD}}=28.16 \mathrm{mN} / \mathrm{m}$. For the particle-liquid interfacial tension, an experimental value is not available, but an estimate can be obtained using the Girifalco and Good mixing rule [30]

$$
\gamma_{S L}=\gamma_{S G}+\gamma_{L G}-2\left(\gamma_{S G} \gamma_{L G}\right)^{1 / 2}
$$

using the octadecene surface tension for the solid-gas interfacial tension $\gamma_{S G}$ and the measured liquid-air interfacial tensions $\gamma_{L G}$. For all liquid compositions used in this work, this estimate yields $\gamma_{S L}<0.1 \mathrm{mN} / \mathrm{m}$, so that $\Delta \gamma \approx$ $28 \mathrm{mN} / \mathrm{m}$, independent of solvent composition. This theoretical prediction has the correct order of magnitude but fails to describe our observations quantitatively, as it would lead to an adsorption free energy of only $-3 k_{B} T$ in the case of pure decalin, compared to $-9 k_{B} T$ found by cryogenic tomography [see the minima in Fig. 3(a)]. A more 
(a)

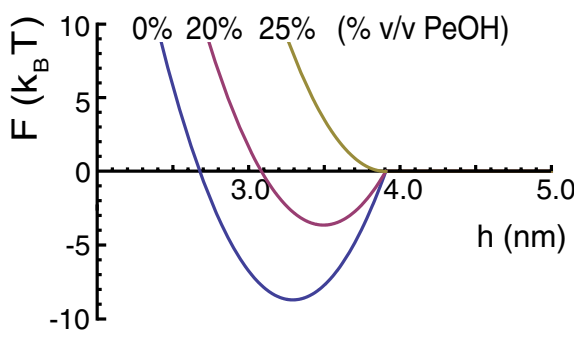

(b)

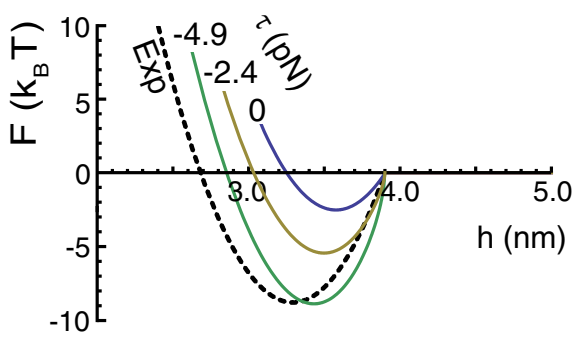

FIG. 3 (color online). (a) Adsorption energy of nanoparticles as a function of distance to the interface at different pentanol concentrations, corresponding to the fitted profiles in Figs. 1(g)-1(i). (b) The effect of line tension $(\tau=0,-2.4$, and $-4.9 \mathrm{pN}$ ) in the case of pure decalin, using $\Delta \gamma=28.1 \mathrm{mN} / \mathrm{m}$.

complete description of the interfacial energies would have to take into account more contributions than the terms of the Pieranski equation. The effects of line tension and net charge on the nanocrystals will now be briefly discussed.

In contrast to microparticles, nanoparticles are expected to exhibit much stronger line tension effects, which cause an additional contribution to the adsorption energy $\Delta F_{\tau}(h)=\tau\left(R^{2}-h^{2}\right)^{1 / 2}$ [31]. As shown in Fig. 3(b), a line tension of $-5 \times 10^{-12} \mathrm{~N}$ is already sufficient to change the adsorption free energy minimum from $-3 k_{B} T$ to $-9 k_{B} T$. This value is close to that reported by Wi et al. [11] and well within the range of $10^{-12}$ to $10^{-6} \mathrm{~N}$ reported experimentally [31]. For models that include a line tension contribution, a discontinuous wetting transition is predicted [31], which can explain the sudden transition from adsorption to desorption of the nanoparticles when the solvent is changed from $20 \%$ to $25 \%$ pentanol. A more systematic study of the dependence of the adsorption energy on particle size would be necessary to determine the magnitude of the line tension.

Electric charging of the particles is expected to play only a minor role. In pure decalin, only $1 \%$ of the particles is expected to be charged, considering the dielectric constants of the particle and the liquid [32]. The dielectric constant increases from 2.1 in pure decalin to 3.1 in $25 \%$ $v / v$ pentanol, resulting in fewer than $2 \%$ of charged particles in our most polar sample.

Although the particle adsorption at the liquid-air interface can be accounted for on the basis of interfacial tensions and line tension, the question remains why the macroscopic measurements of the surface tensions show a qualitatively different behavior. The high adsorption density of $4 \times 10^{17}$ particles $/ \mathrm{m}^{2}$ calculated from Fig. 2 corresponds to 1 particle per $3 \mathrm{~nm}^{2}$, much less than the cross section of the nanoparticle cores $\left(22 \mathrm{~nm}^{2}\right)$. Although multilayer adsorption of nanoparticles has been found in simulations of liquid-liquid interfaces [33], our electron tomographic images show no evidence of multilayer adsorption. Therefore, the species that adsorb to the liquid-air interface and affect the measured interfacial tension are apparently not the nanoparticles themselves. One candidate would be oleic acid molecules desorbed from the surface of nanoparticles, molecules that are about $2 \mathrm{~nm}$ in length. However, oleic acid molecules in aprotic solvents are present as linear apolar dimers [34], which do not adsorb to the liquid-air interface; we verified that the surface tension of decalin remains unchanged (within $0.2 \mathrm{mN} / \mathrm{m}$ ) after dissolution of pure oleic acid, even up to a concentration of $0.040 \mathrm{~mol} / \mathrm{l}$, twice as much as present on the particles (assuming an adsorption density of $\sim 3 \mathrm{~nm}^{-2}$ [35]) in the sample with the highest nanoparticle concentration $(\phi=0.01)$. A more plausible adsorbing species is lead oleate, consisting of a $\mathrm{Pb}^{2+}$ cation coordinated by two oleate ions and being one of the chemical precursors present during nanocrystal growth. We measured that $11 \mu \mathrm{mol} / 1$ lead oleate suffices to decrease the interfacial tension of decalin by $3 \mathrm{mN} / \mathrm{m}$, a concentration which can easily be present in the solution, even after repeated washing of the freshly synthesized nanoparticles. For further proof of this explanation, we measured the interfacial tension of the sample with $30 \% v / v$ pentanol and a nanoparticle volume fraction of $3 \times 10^{-3}$ after sedimenting the nanoparticles by approximately $8 \mathrm{~h}$ centrifugation at $16000 \mathrm{~g}$. The interfacial tension of the supernatant of this centrifuged sample is $26.7 \pm$ $0.2 \mathrm{mN} / \mathrm{m}$, which is within error equal to that of the same sample before centrifugation $(26.5 \pm 0.2 \mathrm{mN} / \mathrm{m})$.

In conclusion, we have demonstrated with cryogenic electron tomography that colloidal nanocrystals coated with oleic acid molecules adsorb strongly at the surface of an apolar liquid and that they desorb when a liquid of lower interfacial tension is added; in contrast, no change in adsorption behavior was evidenced by measurements of the macroscopic surface tension as a function of the concentration of the nanocrystals. Those measurements were dominated by surface-active molecular species. Similar behavior is expected for many other types of nanocrystals, whose surfaces are generally coated with surfaceactive molecules so that they can be dispersed in apolar liquids [36].

Rob Kortschot is thanked for the measurements of the dielectric constants. Remco Tuinier and Daniël Vanmaekelbergh are thanked for useful discussions. This work is part of the research program of the Foundation for Fundamental Research on Matter (FOM), which is part of the Netherlands Organisation for Scientific Research (NWO). 
*Corresponding author.

B.H.Erne@uu.nl

[1] B. P. Binks, Curr. Opin. Colloid Interface Sci. 7, 21 (2002).

[2] E. Vignati, R. Piazza, and T. P. Lockhart, Langmuir 19, 6650 (2003).

[3] Y. Lin, H. Skaff, T. Emrick, A. D. Dinsmore, and T. P. Russell, Science 299, 226 (2003).

[4] T. N. Hunter, R. J. Pugh, G. V. Franks, and G. J. Jameson, Adv. Colloid Interface Sci. 137, 57 (2008).

[5] A. Böker, J. He, T. Emrick, and T. P. Russell, Soft Matter 3, 1231 (2007).

[6] A. Dong, J. Chen, P. M. Vora, J. M. Kikkawa, and C. B. Murray, Nature (London) 466, 474 (2010).

[7] W. H. Evers, B. Goris, S. Bals, M. Casavola, J. de Graaf, R. v. Roij, M. Dijkstra, and D. Vanmaekelbergh, Nano Lett. 13, 2317 (2013).

[8] Y. Lin, A. Böker, H. Skaff, D. Cookson, A. D. Dinsmore, T. Emrick, and T. P. Russell, Langmuir 21, 191 (2005).

[9] S. Ferdous, M. A. Ioannidis, and D. Henneke, J. Nanopart. Res. 13, 6579 (2011).

[10] S. Kutuzov, J. He, R. Tangirala, T. Emrick, T. P. Russell, and A. Böker, Phys. Chem. Chem. Phys. 9, 6351 (2007).

[11] H. S. Wi, S. Cingarapu, K. J. Klabunde, and B. M. Law, Langmuir 27, 9979 (2011).

[12] K. Du, E. Glogowski, T. Emrick, T. P. Russell, and A. D. Dinsmore, Langmuir 26, 12518 (2010).

[13] M. Paulus, P. Degen, S. Schmacke, M. Maas, R. Kahner, B. Struth, M. Tolan, and H. Rehage, Eur. Phys. J. Special Topics 167, 133 (2009).

[14] L. N. Arnaudov, O. J. Cayre, M. A. Cohen Stuart, S. D. Stoyanov, and V. N. Paunov, Phys. Chem. Chem. Phys. 12, 328 (2010).

[15] L. Isa, F. Lucas, R. Wepf, and E. Reimhult, Nat. Commun. 2, 438 (2011)

[16] J. Dubochet, M. Adrian, J. J. Chang, J. C. Homo, J. Lepault, A. W. McDowall, and P. Schultz, Q. Rev. Biophys. 21, 129 (1988).

[17] P. M. Frederik, K. N. Burger, M.C. Stuart, and A.J. Verkleij, Biochim. Biophys. Acta 1062, 133 (1991).

[18] H. Friedrich, P. M. Frederik, G. De With, and N. A. J. M. Sommerdijk, Angew. Chem., Int. Ed. 49, 7850 (2010).
[19] K. Butter, P. H. H. Bomans, P. M. Frederik, G. J. Vroege, and A. P. Philipse, Nat. Mater. 2, 88 (2003).

[20] M. Klokkenburg, R. P. A. Dullens, W. K. Kegel, B. H. Erné, and A.P. Philipse, Phys. Rev. Lett. 96, 037203 (2006).

[21] M. Klokkenburg, B.H. Erné, J.D. Meeldijk, A. Wiedenmann, A. V. Petukhov, R. P. A. Dullens, and A.P. Philipse, Phys. Rev. Lett. 97, 185702 (2006).

[22] J. van Rijssel, B. H. Erné, J. D. Meeldijk, M. Casavola, D. Vanmaekelbergh, A. Meijerink, and A. P. Philipse, Phys. Chem. Chem. Phys. 13, 12770 (2011).

[23] P. A. Midgley, E. P. W. Ward, A. B. Hungría, and J. M. Thomas, Chem. Soc. Rev. 36, 1477 (2007).

[24] M. V. Kovalenko, D. V. Talapin, M. A. Loi, F. Cordella, G. Hesser, M. I. Bodnarchuk, and W. Heiss, Angew. Chem., Int. Ed. 47, 3029 (2008).

[25] See Supplemental Material at http://link.aps.org/ supplemental/10.1103/PhysRevLett.111.108302 for details of the particle synthesis, tomographic dataset movies, and evidence of the colloidal stability of the studied liquid dispersions of nanoparticles.

[26] J. R. Kremer, D. N. Mastronarde, and J. R. McIntosh, J. Struct. Biol. 116, 71 (1996).

[27] C. B. Murray, D. J. Norris, and M. G. Bawendi, J. Am. Chem. Soc. 115, 8706 (1993).

[28] R. J. Hunter, Introduction to Modern Colloid Science (Oxford University Press, New York, 1993), 1st ed.

[29] P. Pieranski, Phys. Rev. Lett. 45, 569 (1980).

[30] L. A. Girifalco and R. J. Good, J. Phys. Chem. 61, 904 (1957).

[31] F. Bresme and M. Oettel, J. Phys. Condens. Matter 19, 413101 (2007).

[32] M. Shim and P. Guyot-Sionnest, J. Chem. Phys. 111, 6955 (1999).

[33] R. J. K. U. Ranatunga, C. T. Nguyen, B. A. Wilson, W. Shinoda, and S. O. Nielsen, Soft Matter 7, 6942 (2011).

[34] D. H. Lee and R. A. Condrate, J. Mater. Sci. 34, 139 (1999).

[35] M. Klokkenburg, J. Hilhorst, and B.H. Erné, Vib. Spectrosc. 43, 243 (2007).

[36] C. Murray, C. Kagan, and M. Bawendi, Annu. Rev. Mater. Sci. 30, 545 (2000). 